\title{
¿Cómo interfiere la ansiedad, la probabilidad de actuación y la defensa de los propios derechos en alumnos con dificultades de aprendizaje en su rendimiento?
}

\author{
Deilis-Ivonne Pacheco, $\mathrm{M}^{\mathrm{a}}$ del Carmen Díez y Jesús-Nicasio García \\ Universidad de León (España)
}

\begin{abstract}
Para el ajuste social correcto, los iguales son agentes cruciales, tanto psicoemocional como socioeducativo y conductual de los alumnos, ya que se trata de figuras con perspectivas similares y que favorecen la adquisición de habilidades sociales, lazos de amistad, adaptación a nuevos roles sociales etc. La ansiedad, probabilidad de actuación y la defensa de los propios derechos interfieren en alumnos con dificultades de aprendizaje, ya que carecen de habilidades sociales no sólo para percibir y comprender las relaciones lógicas, sino también para prevenir y escoger el comportamiento adecuado en situaciones sociales. Por ello, el objetivo de la presente comunicación es analizar las diferentes variables de las habilidades sociales en alumnos con dificultades de aprendizaje. La muestra para llevar a cabo el estudio corresponde a 193 alumnos de $4^{\circ}$ a $6^{\circ}$ de educación primaria de diversa tipología (alumnos con y sin dificultades de aprendizaje (DA, sDA) y alumnos con déficit de atención e hiperactividad (TDAH). Los resultados que se obtiene muestran una desventaja emocional en relación a los alumnos DA y TDAH en relación a los alumnos sin dificultades de aprendizaje (SDA).
\end{abstract}

Palabras clave: Autocontrol, defensa de los propios derechos, ansiedad, dificultades de aprendizaje, relaciones, adaptación.

How different variables interfere with social skills in students with learning disabilities? To correct social setting, peers are key actors, both as a socio-psycho-emotional and behavioral students since figures is similar perspectives and promote the acquisition of social skills, friendships, adapting to new social roles etc. Anxiety, likelihood of action and defense of the rights themselves interfere with students with learning disabilities because they lack social skills not only to perceive and understand the logical relationships, but also to prevent and choose the appropriate behavior in social situations. Therefore, the purpose of this communication is to analyze the different variables of social skills in students with learning disabilities. The sample for carrying out the study corresponds to 193 students from 4th to 6th year of primary education of different type (children with and without learning disabilities and students with attention deficit hyperactivity disorder (ADHD). The results obtained show an emotional disadvantage in relation to LD and ADHD students compared to students without learning disabililties.

Key words: Self-control, defense of the rights themselves, anxiety, learning disabilities, relationships, adaptation.

Correspondencia: Jesús Nicasio García Sánchez. Universidad de León. Departamento de Psicología, Sociología y Filosofía. Area de Psicología Evolutiva y de la Educación, Campus de Vegazana, s/n, C.P. 24071. León (España).E-mail: jn.garcia@unileon.es 
En general, los estudios empíricos revisados destacan las habilidades sociales de asertividad, cooperación y autocontrol, resaltando los problemas de estas habilidades en los alumnos con dificultades de aprendizaje y/o bajo rendimiento y/o fracaso escolar y que además, carecen de habilidades sociales no sólo para percibir y comprender las relaciones lógicas y causales que implica la cognición social, sino también para prevenir y escoger el comportamiento adecuado en situaciones sociales (Bauminger, Morash y Schorr, 2005; Pan-Skadden et al., 2009).

En función de los datos revisados, nos parece relevante analizar habilidades tales como el autocontrol y la cooperación ya que son habilidades importantes desde el punto de vista del profesor para fomentar y conseguir un buen clima en el aula (Díez, García y Pacheco, 2007a; Meier, Diperna y Oster, 2006; Miltenberger, 2008) así como la asertividad, las habilidades de interacción y las habilidades de conversación, etc. (Martorell, González, Rasal y Estellés (2009). Ya que consideramos, según los estudios revisados, que dichos alumnos presentan carencias y limitaciones en las mismas y por ello nuestro estudio incide no sólo en un mero estudio teórico, sino en un análisis desde el punto de vista psicológico de tales habilidades, a partir de unos instrumentos previamente elaborados para tales fines.

Por todo ello, el objetivo de este estudio se centró, en las habilidades sociales que influyen en el desarrollo y aprendizaje de los alumnos con dificultades de aprendizaje y/o bajo rendimiento y/o fracaso escolar, específicamente relacionado con la composición escrita.

Debido a las limitaciones que presentan los alumnos con dificultades de aprendizaje en las habilidades sociales, se debería mostrar la importancia de sensibilizar a los profesores y estudiantes, para así poder integrar sentimientos (competencia emocional), pensamientos (competencia cognitiva) y acciones (competencia del comportamiento) y de este modo promover el ajuste, esfuerzo y motivación (Lackaye y Margalit, 2006; Megan y Christine, 2008). Teniendo en cuenta estos aspectos, proponemos una evaluación del alumno tanto desde el punto de vista del mismo como del profesor.

\section{Instrumento dirigido al alumno (HHSS/A)}

Teniendo en cuenta todas estas aportaciones y con el propósito de valorar las habilidades sociales del alumno, se diseñaron los instrumentos de autoinforme sobre el contenido emocional. El despliegue de las habilidades sociales (EM), el inventario de ansiedad en situaciones de despliegue de las habilidades anteriormente referenciadas (AN), así como el inventario de probabilidades de actuación en las situaciones descritas anteriormente (AC), (Díez, García y Pacheco, 2007b; 2007c; 2008).

Otro dato de interés para el estudio es el grado de ansiedad y probabilidad con la que el alumno realiza las diversas conductas. Según los estudios revisados, tales alumnos presentan un alto grado de ansiedad y baja probabilidad de actuación. Por ello, 
nos ha parecido relevante construir el instrumento que mide la ansiedad (AN) y el instrumento (AC) que nos aporta información sobre la probabilidad de actuación del alumno y que conjuntamente podamos obtener el perfil de una persona.

Por todo ello, se pretende medir y analizar en una muestra representativa de alumnos de Educación Primaria cómo influyen las habilidades sociales en los alumnos con/sin dificultades de aprendizaje y en qué tipo de habilidades presentan déficits tales alumnos, todo ello relacionado con la composición escrita.

\section{METODO}

\section{Participantes}

La muestra para llevar a cabo el estudio se realizó con un total de 462 alumnos y alumnas con edades comprendidas entre 9 y 12 años, que cursaban estudios desde $4^{\circ}$ a $6^{\circ}$ escolarizados en Educación Primaria.

Se obtuvo una muestra de alumnos de segundo y tercer ciclo de Educación Primaria. La misma fue seleccionada en función de varios criterios para poder así analizar las habilidades sociales de los alumnos con/sin dificultades de aprendizaje.

El primer criterio, está basado en la evaluación de los alumnos que habían sido examinados en estudios previos. De esta manera se seleccionaron tanto a alumnos como a profesores. El estudio se realizó de forma independiente por dos investigadoras del equipo, de tal forma que no interfiriera en los resultados. Esto aseguró la fidelidad de los objetivos, puesto que los profesores desconocían la investigación, con el fin de que no existieran sesgos en la misma.

El segundo criterio consistió en seleccionar a alumnos de diferentes tipologías, es decir, alumnos con DA, sin DA y con TDAH. Estos últimos, procedían de la asociación de padres de TDAH de la Provincia de León ALENHI (Asociación Leonesa de Afectados por el Trastorno de Déficit de Atención con y sin Hiperactividad). Se puede ver con más detalle en la figura 1.

Figura 1. Muestra de alumnos en función del nivel y tipología

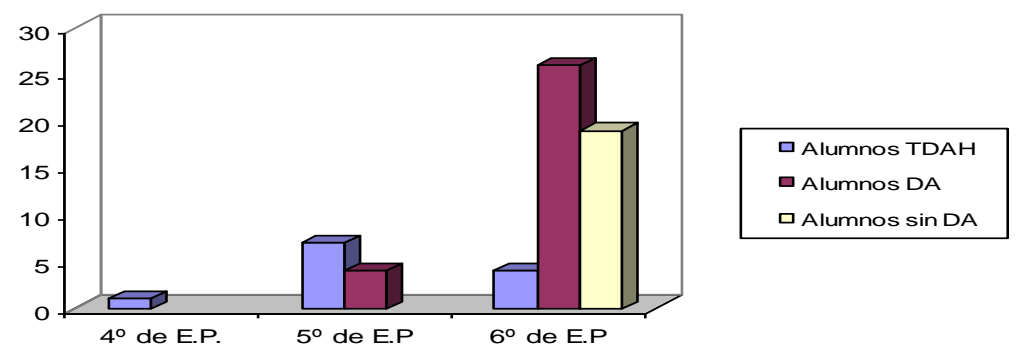

Aplicando los dos criterios, se seleccionó la muestra de 61 alumnos de $4^{\circ}$ a $6^{\circ}$ que están clasificados en función de diferente tipología: 12 alumnos con TDAH, 30 con 
DA y 29 SDA (de los cuales el $36 \%$ mujeres y el 64\% hombres, la mayoría el 98\% cursan como lengua extranjera el inglés, el $45 \%$ cursan estudios en escuelas públicas).

A continuación, en la tabla 1 se recoge la muestra de alumnos en función del género y ciclo educativo.

Tabla 1. Distribución de la muestra de alumnos en función de la tipología, género, edad y ciclo educativo

\begin{tabular}{|c|c|c|c|c|c|c|c|c|c|}
\hline \multirow{2}{*}{ Tipo de Alumnos } & \multicolumn{2}{|c|}{$4^{\circ}$ de E.P. } & \multicolumn{2}{|c|}{$5^{\circ}$ de E.P } & \multicolumn{2}{|c|}{$6^{\circ}$ de E.P } & \multicolumn{2}{|c|}{ Total género/grupo } & \multirow{2}{*}{$\begin{array}{l}\text { Total } \\
\text { grupo }\end{array}$} \\
\hline & Hombres & Mujeres & Hombres & Mujeres & Hombres & Mujeres & Hombres & Mujeres & \\
\hline Alumnos TDAH & 1 & & 7 & & 3 & 1 & 11 & 1 & 12 \\
\hline Alumnos DA & & & 2 & 2 & 16 & 10 & 18 & 12 & 30 \\
\hline Alumnos sin DA & & & & & 11 & 8 & 11 & 8 & 19 \\
\hline Total Género & 1 & 0 & 9 & 2 & 30 & 19 & 40 & 21 & 61 \\
\hline Total Nivel & \multicolumn{2}{|c|}{1} & \multicolumn{2}{|c|}{11} & \multicolumn{2}{|c|}{49} & \multicolumn{2}{|c|}{61} & \\
\hline
\end{tabular}

\section{Instrumentos}

Los instrumentos que se utilizaron para medir las variables de los alumnos relacionadas con las habilidades sociales se exponen a continuación:

Tabla 2. Aspectos evaluados e instrumentos utilizados para la investigación

\begin{tabular}{lccc}
\hline \multicolumn{1}{c}{ Aspectos evaluados } & Instrumento & Tareas & Parámetros \\
\hline Escala-Valoración & & Maestros & \\
\hline & HHSS/VP & Cuestionario tipo Líkert & Puntuación \\
\hline Autoinforme-Emocional & & Alumnos & \\
\hline Inventario-Ansiedad & HHSS/EM & Cuestionario tipo Líkert & Puntuación \\
\hline Inventario-Probabilidad de actuación & HHSS/AN & Cuestionario tipo Líkert & Puntuación \\
\hline
\end{tabular}

Tales instrumentos, fueron diseñados con el propósito de apreciar los aspectos emocionales de los alumnos, tales como, el autocontrol, la defensa de sus propios derechos, las habilidades de conversación, etc., ya que la mayoría de las investigaciones destacan que los alumnos con dificultades en el desarrollo presentan dificultades en las habilidades sociales. Con todo ello, se pretende detectar y contrastar las respuestas del alumno (HHSS /A) con las del profesor (HHSS /P) y obtener medidas válidas y fiables tanto desde el punto de vista del alumno como del profesor con el propósito de evaluar las habilidades sociales de los niños de educación primaria.

Así el instrumento (HHSS/A) está formado por distintas partes: la primera, se centra en evaluar las habilidades sociales desde el punto de vista emocional (EM); la segunda, las habilidades sociales desde el punto de vista de la ansiedad (AN) y por último, la probabilidad de actuación (AC).

\section{Procedimiento}

La investigación que se presenta, se inició, por una parte, con la revisión de las bases de datos en Academia Search Elite y, concretamente, con la revisión y análisis 
de los estudios empíricos que se encuentran relacionados con el tema de estudio. Por otro lado, con la revisión de los Reales Decretos, Programas y Guías educativas donde se establece el currículo de Educación Primaria, con el fin de obtener datos tales como el rendimiento, que se encuentran relacionados con el foco de estudio de la presente investigación.

A partir de aquí, se construyó el Instrumento (HHSS/A) que está formado por varias subescalas: la primera, se centra en evaluar las habilidades sociales desde el punto de vista emocional (EM); la segunda, las habilidades sociales desde el punto de vista de la ansiedad (AN) y por último, la probabilidad de actuación (AC).

A continuación, se ha llevado a cabo, el diseño y plan de muestreo de forma experimental en un centro, que permitiera así verificar el tiempo de aplicación de este Protocolo en los centros educativos de la provincia de León. Seguidamente, se procedió al muestreo a partir de diversos colegios que nos permitieran así seleccionar un grupo representativo de alumnos objeto de estudio. Para ello, se necesitó la colaboración de los centros educativos, profesorado, etc. cuyo criterio de selección fue escoger niños/as con bajo rendimiento y/o dificultades de aprendizaje y/o fracaso escolar.

La recogida de la muestra se realizó directamente por las investigadoras y no por otros medios, con el fin de asegurar la fiabilidad y validez de los instrumentos en la recogida de información. Una vez realizado el trabajo de campo y recogidos todos los protocolos, en total de 462, se procedió a la codificación e informatización de los datos en la matriz Excel y la interpretación de los resultados.

\section{RESULTADOS}

Para analizar la relación de los alumnos con dificultades de aprendizaje y sus habilidades sociales, se realizaron los contrastes multivariados en las medidas de los alumnos en función de su percepción de las notas de lengua (Alto, Bajo, Medio, Muy alto), que indican resultados altamente significativos y con un tamaño del efecto pequeño $\left[F_{(40.1959)}=2.887, p=.001, \eta^{2}=.049\right]$.

Para la realización de estos análisis multivariados se tomaron como factores intersujetos las medidas de los alumnos en función de su percepción de la nota de lengua (Alto, Bajo, Medio, Muy alto); y, como variables dependientes, las diferentes medidas obtenidas de los alumnos. Esto se hizo con el fin de poner en evidencia en qué medida las habilidades sociales de los alumnos son diferentes en función de la percepción de la nota, para intentar comprender la relación entre la percepción de los alumnos en función de su rendimiento en lengua y las habilidades sociales de los mismos.

Las pruebas de los efectos intersujetos o entre grupos señalan, igualmente, resultados muy significativos estadísticamente en la mayoría de las variables dependientes relativas a las habilidades sociales de los alumnos. Para ver en detalle estos resultados. 
Al tomar como variable de agrupamiento (factor inter-sujetos) la pertenencia a un grupo de alumnos de percepción de la nota de lengua (muy alto, alto, medio y bajo), se observan diferencias estadísticamente significativas sobre todo en el grupo de percepción alta y baja en las habilidades sociales.

Se observan diferencias significativas en variables de las habilidades sociales, pero sobre todo en los aspectos emocionales así como en las variables conductuales centradas en la probabilidad de actuación. Por ejemplo, la toma de conciencia y control emocional $\left[F=10.23, p=. \quad 001 ; \eta^{2}=.101\right)$, las habilidades de conversación $\left[F={ }_{75.2}, p=\right.$. 001; $\left.\eta^{2}=.076\right)$, las habilidades socio-emocionales $\left[F={ }_{9.99}, p=.001\right.$; $\left.\eta^{2}=.099\right)$, las habilidades de cooperación y ayuda durante el trabajo $[F=8.77 p=.001$; $\left.\eta^{2}=.088\right)$ y en las en las habilidades sociales-aspecto emocional en las habilidades de vida y de bienestar subjetivo se obtienen resultado estadísticamente significativos $\left[F=2.12, p=.001 ; \eta^{2}=.085\right)$. A excepción de las habilidades-aspecto emocional en la defensa de los propios derechos y en las habilidades conductuales-probabilidad de actuación donde no se observan diferencias estadísticamente significativas. Se puede ver con más detalle en la figura 2.

Figura 2. Medias de las variables analizadas en el estudio

$\square M U Y$ ALTO $(N=88)$
$\square A L T O(N=199)$
$\square M E D I O(N=128)$
$\square B A J O(N=37)$

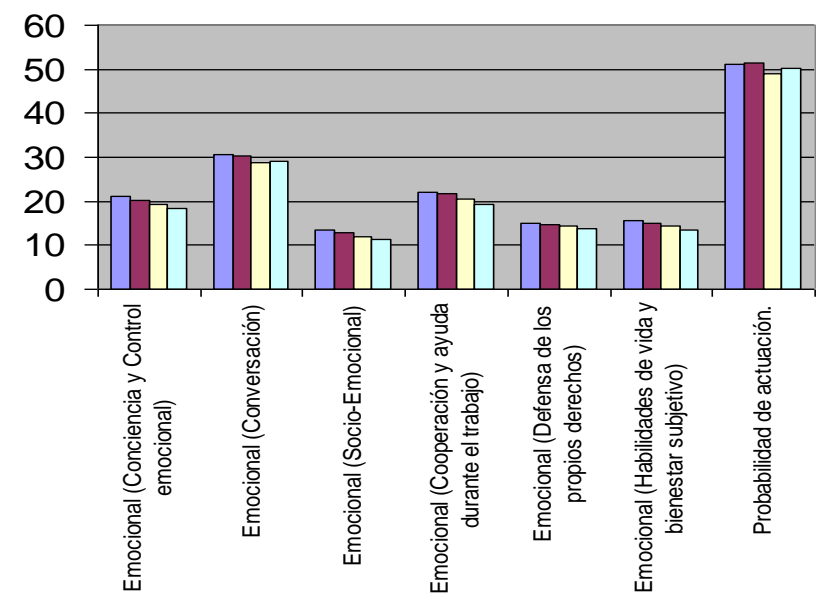

Cuando se cotejan los contrastes post hoc entre las variables estadísticamente significativas obtenidas en las pruebas de los efectos intersujetos (entre los grupos) encontramos diferencias estadísticas significativas en muchas de ellas, como puede verse en la figura 2. Por ejemplo, hay una tendencia general en el grupo de percepción alta frente a medio y bajo. Por ejemplo, se observan diferencias significativas en los contrastes post hoc entre los grupos en las medidas muy alto y bajo-medio (nivel de percepción) en la habilidades de conciencia y control emocional (ej., $\mathbf{M}_{\text {Muyalto }}=21.19$. vs., 
$\mathbf{M}_{\text {bajo }}={ }_{18.52}=.001$ ), en los grupos (ej., $\mathbf{M}_{\text {Muyalto }}={ }_{21.19}$. vs., $\mathbf{M}_{\text {medio }}={ }_{19.39}=.001$ ) excepto entre los grupos de medidas Alto vs., Medio que no se encuentran diferencias estadísticamente significativas.

Este patrón se observa también en las variables que hacen referencia a las Habilidades de vida y bienestar subjetivo, en los grupos muy alto/bajo (ej., $\mathbf{M}_{\text {Muyalto }}=21.19$ , vs., $\mathrm{M}_{\text {bajo }}={ }_{18.52}=.001$ ), en los grupos muy alto/medio (ej., $\mathbf{M}_{\text {Muyalto }}=15.73$, vs., $\left.\mathrm{M}_{\text {medio }}={ }_{14.35}=.009\right)$, y en las habilidades que hacen referencia a los aspectos socioemocionales, en los grupos muy alto/bajo (ej., $\mathbf{M}_{\text {Muyalto }}=_{13.40}$. vs., $\mathbf{M}_{\text {bajo }}={ }_{11.39}=.004$ ), en los grupos muy alto/medio (ej., $\mathbf{M}_{\text {Muyalto }}={ }_{13.40}$. vs., $\mathbf{M}_{\text {medio }}={ }_{11.99}=.002$ ).

En cuanto a las Habilidades de conversación, presentan diferencias estadísticamente significativas entre los grupos alto/medio (ej., $\mathbf{M}_{\text {alto }}={ }_{30.31}$ vs., $\mathrm{M}_{\text {medio }}={ }_{28.91}=.073$ ) $\mathrm{y}$ entre los grupos muy alto/medio (ej., $\mathbf{M}_{\text {Muyalto }}=30.61$., $\mathrm{M}_{\text {medio }}={ }_{28.91=.066)}$.

En el caso de las habilidades de cooperación y ayuda durante el trabajo, podemos ver cómo aparecen diferencias estadísticamente significativas entre los grupos alto/bajo (ej., $\mathbf{M}_{\text {alto }}={ }_{14.60}$. vs., $\mathbf{M}_{\text {bajo }}={ }_{13.67}=.009$ ), en los grupos muy alto/bajo (ej., $\mathrm{M}_{\text {Muyalto }}={ }_{15.02}$. vs., $\mathrm{M}_{\text {bajo }}={ }_{13.67}=.002$ ).

Otros patrones de interés, por ejemplo, en el caso de la defensa de los propios derechos (habilidad socio-emocional) y en las habilidades conductuales (probabilidad de actuación) no se observan diferencias estadísticamente significativas.

Tabla 3. Contrastes post hoc que son significativos, en los análisis multivariados, tanto para la percepción de la nota de lengua (alto, bajo, medio) como para las para las medidas de habilidades sociales

\begin{tabular}{|c|c|c|c|c|}
\hline \multirow[b]{2}{*}{ Variables } & \multicolumn{4}{|c|}{ Percepción nota de lengua } \\
\hline & $\begin{array}{l}\text { ALTO vs. } \\
\text { BAJO }\end{array}$ & $\begin{array}{l}\text { ALTO vs. } \\
\text { MEDIO }\end{array}$ & $\begin{array}{l}\text { MUY ALTO } \\
\text { vs. BAJO }\end{array}$ & $\begin{array}{l}\text { MEDIO vs. } \\
\text { MUY ALTO }\end{array}$ \\
\hline $\begin{array}{l}\text { Habilidades sociales -Emocional (Conciencia y } \\
\text { Control emocional) }\end{array}$ & .042 & n. s. & .001 & .001 \\
\hline Habilidades sociales -Emocional (Conversación) & n.s. & .073 & n.s. & .066 \\
\hline $\begin{array}{llll}\text { Habilidades } & \text { sociales } & \text {-Emocional } & \text { (Socio- } \\
\text { Emocional) } & & & \end{array}$ & .094 & n.s. & .004 & .002 \\
\hline $\begin{array}{l}\text { Habilidades sociales -Emocional (Cooperación y } \\
\text { ayuda durante el trabajo) }\end{array}$ & .009 & .083 & .002 & .024 \\
\hline $\begin{array}{l}\text { Habilidades sociales -Emocional (Defensa de los } \\
\text { propios derechos) }\end{array}$ & n.s. & n.s. & n.s. & n.s. \\
\hline $\begin{array}{l}\text { Habilidades sociales -Emocional (Habilidades } \\
\text { de vida y bienestar subjetivo) }\end{array}$ & .022 & n.s. & .001 & .009 \\
\hline Habilidades sociales -Probabilidad de actuación. & n.s. & n.s. & n.s. & n.s. \\
\hline
\end{tabular}

\section{DISCUSION}

El objetivo del presente estudio se centra analizar cómo son las habilidades sociales de los alumnos con dificultades de aprendizaje y/o bajo rendimiento. 
Para el estudio, se diseño el Protocolo Habilidades Sociales en los alumnos de Educación Primaria (HHSS), elaborado para tal efecto y compuesto por un conjunto de cuatro escalas que permitan valorar las habilidades sociales desde el punto de vista del alumno, los aspectos emocionales a través de HHSS/EM, los aspectos fisiológicos de los alumnos a través del HHSS/AN que evalúan el grado de ansiedad del sujeto, el grado de probabilidad de actuación con HHSS/AC.

Como aportación de la presente investigación, el hecho de tomar como factores intersujetos los cuatro tipos de percepción de la nota de lengua (Muy alto, alto, medio y bajo) y como variables dependientes las diferentes medidas obtenidas de los alumnos en cuanto a sus habilidades sociales, los resultados obtenidos son muy significativos estadísticamente en la mayoría de las variables dependientes relativas a los alumnos. En cuanto a los alumnos, al tomar como variable de agrupamiento la percepción de la nota, se observaron diferencias estadísticamente significativas, sobre todo en variables que indican aspectos emocionales. Por ejemplo, se observan diferencias significativas en variables que hacen referencia a las habilidades de vida y bienestar subjetivo, a la conciencia y control emocional, a las habilidades de cooperación y ayuda durante el trabajo y las habilidades de conversación.

Otros patrones de interés, por ejemplo, en el caso de la defensa de los propios derechos (habilidad socio-emocional) y en la probabilidad de actuación, podemos ver cómo no aparecen diferencias significativas entre los grupos, según la percepción de la nota de lengua de los alumnos.

Por otra parte, se intenta predecir las habilidades emocionales de los alumnos en función de la percepción de los mismos a través de las notas de lengua, podemos concluir diciendo que, la mayoría de los alumnos presentan una claros y adecuados comportamientos conductuales (Habilidades de actuación); sin embargo, podemos notar una clara desventaja en habilidades emocionales desde el punto de vista cognitivoemocional.

Teniendo en cuenta estas aportaciones, debemos enumerar una serie de limitaciones y perspectivas futuras que esperamos superar en el siguiente estudio empírico. Aplicando el estudio en una muestra más representativa queremos corroborar o en su defecto subsanar los resultados. En primer lugar, analizar la relación entre las habilidades sociales y las dificultades de aprendizaje en alumnos de diferente tipología (DA, sDA y TDAH); en segundo lugar, aplicando el estudio a una muestra más amplia de alumnos de Educación Primaria se pretende analizar la influencia de las habilidades sociales tanto a nivel socio-emocional como en su repercusión en el rendimiento del área de lengua, concretamente en la escritura.

Por otra parte, sería deseable la participación activa de los profesores y su implicación, a través de las diversas materias y áreas para el desarrollo de las HHSS en los alumnos, puesto que sabemos que la creencia de los profesores en su propia 
capacidad para ser eficientes en la instrucción afecta los resultados del aprendizaje y del rendimiento de los alumnos (Pacheco, García y Díez, 2009).

\section{Agradecimientos}

Durante la realización de este estudio se recibieron ayudas competitivas del proyecto del MICINN (EDU2010-19250) y con fondos FEDER de la Unión Europea para el trienio 2010-2013; así como del proyecto al Grupo de Investigación de Excelencia de la JCyL GR-259; (BOCyL 27 de abril de 2009) para el trienio 2009-2011. Proyectos concedidos al IP/director J N García.

\section{REFERENCIAS}

Bauminger, H., Morash, J. y Schorr, N. (2005). Social Information Processing and Emotional Understanding in Children with LD. Journal of Learning Disabilities, 38(1), 45-61.

Díez, C., García, J.N. y Pacheco, D.I. (2007a). Habilidades Sociales en los alumnos con dificultades en el desarrollo. En J.N. García (Coord.), Dificultades del desarrollo: Evaluación e intervención (pp. 229-236). Madrid: Pirámide.

Díez, C., García, J.N. y Pacheco, D.I. (2007b). Instrumento de evaluación de las habilidades sociales en los alumnos de Educación Primaria con y sin dificultades del desarrollo. Instrumentos y programas de intervención en las dificultades del desarrollo. En J.N. García (Coord.), Dificultades del desarrollo: Evaluación e intervención (CD-ROM). Madrid: Pirámide.

Díez, C., García, J.N. y Pacheco, D.I. (2007c). Instrumento de evaluación de las habilidades sociales valorado por el profesor, en alumnos con y sin dificultades del desarrollo. Instrumentos y programas de intervención en las dificultades del desarrollo. En J.N. García (Coord.,), Dificultades del Desarrollo: Evaluación e Intervención (CD-ROM) Madrid: Pirámide.

Díez, C., Pacheco, D.I. y García, J.N. (2008). Las habilidades sociales en el marco del EEES (pp. 239-247). En M. Hijano (Coord.), Las titulaciones de educación ante el Espacio Europeo de Educación Superior: Análisis de experiencias. Málaga: Aljibe.

Martorell, M., González, R., Rasal, P. y Estellés, R. (2009). Convivencia e inteligencia emocional en niños en edad escolar. European Journal of Education and Psychology, 1(2), 69-78.

Megan, W. y Christine, C. (2008). Social competence and learning difficulties: Teacher perceptions. Therapy Journal, 55(4), 256-265.

Meier, C.R., DiPerna, J.C. y Oster, M.M. (2006). Importance of Social Skills in the Elementary Grades. Education and Treatmente of Children, 29(3), 409-419.

Miltenberger, R.C. (2008). Teaching safety skills to children: Prevention of firearm injury as an exemplar of best practice in assessment, training, and generalization of safety skills. Behavior Analysis in Practice, 1(1), 30-36.

Lackaye, T.D. y Margalit, M. (2006).Comparisons of achievement effort, and self-perceptions among students with learning disabilities and their peers from different achievement groups. Journal of Learning Disabilities, 39(5), 432-446.

Pacheco, D.I., García, J.N. y Díez, C. (2009). Self-efficacy, approach, and teacher's practice in teh writing teaching. European Journal of Education and Psychology, 2(2), 5-23. 
Pan-Skadden, J., Wilder, D.A., Sparring, J., Severtson, E., Donalson, J., Postma, N., Beavers, G. y Neidert, P. (2009). The use of behavioural skills training and in-situ training to teach children to solicit help when lost: A preliminary investigation. Education and Treatment of Children, 32(3), 259-370.

Recibido: 10 de febrero de 2010 Recepción Modificaciones: 16 de abril de 2010 Aceptado: 19 de abril de 2010 